\title{
Étude physico-chimique de sept (7) plantes spontanées alimentaires du centre-ouest de la Côte d'Ivoire.
}

\author{
KOUAME N'dri Marie-Thérèse 1, SORO Kafana 1, MANGARA Ali 1, DIARRASSOUBA Nafan 2, \\ KOULIBALY Annick Victoire ${ }^{3}$ et BORAUD N'Takpé Kama Maxime 4 \\ 1. Centre de Recherche en Écologie de l'Université Nangui-Abrogoua (Côte d'Ivoire) \\ 08 BP 109 Abidjan 08, Côte d'Ivoire.. ndrimaritherese@yahoo.fr, SORO Kafana, skafana1@yahoo.fr, 225084947 39, \\ MANGARA Ali, Mangarali@yahoo.fr, 225, 01083333 \\ 2. Enseignement et de recherche à Sciences biologiques Unité de formation et de recherche / Université de \\ Péléforo Gon Coulibaly Université (PUPTC de Korhogo - Côte d'Ivoire). BP 1328 KORHOGO \\ E mail : nanfandiarra@yahoo.fr \\ 3. Département de l'Agroforesterie,Université Jean Lorougnon Guédé 01 BP 10588 Abidjan 01; Côte d'Ivoire \\ (UJLoG) Daloa, Côte d'Ivoire E mail : koulannick@yahoo.fr \\ $4 . \quad$ Laboratoire de Botanique, U.F.R. Biosciences, Université Félix Houphouët Boigny \\ 22 BP 582 Abidjan 22, Côte d'Ivoire. E mail : boraudn@hotmail.com \\ Auteur correspondant email: ndrimaritherese@yahoo.fr
}

Original submitted in on $8^{\text {th }}$ October 2015. Published online at www.m.elewa.org on $30^{\text {th }}$ June 2015 http://dx.doi.org/10.4314/jab.v90i1.12

\section{RESUME}

Objectif: Une enquête ethnobotanique, visant à inventorier les plantes spontanées alimentaires encore présentes dans la région du Fromager (Centre-Ouest) de la Côte d'Ivoire, a été menée auprès des populations rurales.

Méthodologie et résultats : Parmi ces espèces, sept (7) d'entre elles dont les organes sont les plus consommés par les populations ont fait l'objet d'étude monographique et ont été retenues pour une analyse comparative des nutriments. Ce sont : Irvingia gabonensis (Kaklou), Ricinodendron heudelotii(Apki) Beilschmiedia mannii (Bitéi), Treculia africana (Blenbledou), Myrianthus arboreus (Ticliti), Sesamum indicum (Mahikor), et Strombosia pustulata (Klê-kor). Les résultats des analyses des organes comestibles se répartissent en oléagineuses Irvingia gabonensis (83,51\%.), Ricinodendron heudelotii (58,76 \%.) et Sesamum indicum $(65,26 \%$.), en protéagineuses Beilschmiedia mannii (42,85\%.), Strombosia pustulata (53,03\%.) et Myrianthus arboreus (57,2 $\%$.) et en sucre Treculia africana $(23,12 \%$.). Quant aux feuilles de Myrianthus arboreus, elles ont une teneur très faible en sucre et peuvent être conseillé aux diabétiques.

Conclusion et application des résultats: La connaissance des valeurs nutritionnelles de ces produits contribuera à une meilleure prise de conscience du rôle essentiel que peuvent jouer les plantes spontanées alimentaires afin de pouvoir contribuer à améliorer le bien-être des populations locales en nourriture et en ressources financières.

Mots clés : Plantes spontanées alimentaires, teneur en nutriment, zone forestière, Côte d'Ivoire. 
ABSTRACT

Objective: An ethnobotanical survey, aiming at making a list of wild food plants still present in the "Fromager" region (Central west) of Côte d'Ivoire, was made among the rural populations.

Methodology and results: Seven (7) plants that are the most consumed by the populations were the focus of a monographic study and were selected for a comparative analysis of nutrients. These were: Irvingia gabonensis (Kaklou), Ricinodendron heudelotii(Akpi), Beilschmiedia mannii (Bitéi), Treculia africana (Blenbledou), Myrianthus arboreus(Ticliti), Sesamum indicum (Mahikor) and Strombosia pustulata (Klê-Kor). The results of the analyses of the edible parts was divided up into the oleaginous Irvingia gabonensis $(83.51 \%)$ Ricinodendron heudelotii (58.76\%.) and Sesamum indicum (65.26\%.); proteaginous Beilschmiedia mannii (42.85\%.), Strombosia pustulata $(53.03 \%$.) and Myrianthus arboreus (57.2\%.) and in sugar Treculia africana $(23.12 \%$.). As for the leaves of Myrianthus arboreus, they had a very low content of sugar and can be recommended to diabetics.

Conclusion and application of results: Knowing the nutritional value of these products will contribute to a better awareness of the essential role that wild food plants can play in improving local people welfare in terms of food and financial resources.

Keywords : Forest food sorts species, food values, forest zone, Côte d'Ivoire.

\section{INTRODUCTION}

La Côte d'Ivoire est un réservoir de richesses naturelles (faune, flore, biosphère etc.), disséminées sur toute l'étendue du territoire (Aubreville, 1959).Cependant, au niveau de la connaissance, de la sauvegarde, de la protection et de la valorisation de ces patrimoines, les approches interdisciplinaires qui permettraient une stratégie plus globale sont quasi inexistantes. Malheureusement tout cela a entrainé la diminution de la superficie occupée par ces écosystèmes végétaux. De 15 millions d'ha de forêt en 1960, elle est passée à 2,5 millions d'hatare en 1990 (Aké-Assi et Boni, 1990). Cela a causé la disparition de plusieurs plantes alimentaires spontanées. Ceci a constitué également l'un des principaux facteurs de paupérisation des communautés rurales du fait que Certaines pratiques culturelles et savoir-faire traditionnels sont en voie de disparitions :c'est le cas de , Beilschmiedia mannii, une plante qu'on retrouve à l'ouest de la
Côte d'lvoire dont les graines découpées en morceaux sont séchées et réduites en poudre (Figure 1). La poudre obtenue sert à la préparation d'une sauce gluante, cette sauce se consomme généralement avec le foutou de manioc pendant les cérémonies de réjouissance. Aujourd'hui cette pratique est en train de disparaitre du au faite que les fruits deviennent rare (figure 2). D'où les produits exotiques (gombos, aubergines, tomates, arachides) plus coûteux concurrencent et évincent des aliments traditionnels, perturbant ainsi la production agricole locale, modifiant les habitudes alimentaires et aggravant parfois les déséquilibres nutritionnels. Une meilleure connaissance des aliments locaux, de leurs caractéristiques nutritionnelles et des technologies traditionnelles qui leur sont appliquées est l'un des facteurs qui permettront d'assurer la sécurité alimentaire et culturelle des populations. 


\section{Kouame et al. J. Appl. Biosci. Étude physico-chimique de sept (7) plantes spontanées alimentaires du centre-ouest de la Côte d'Ivoire}

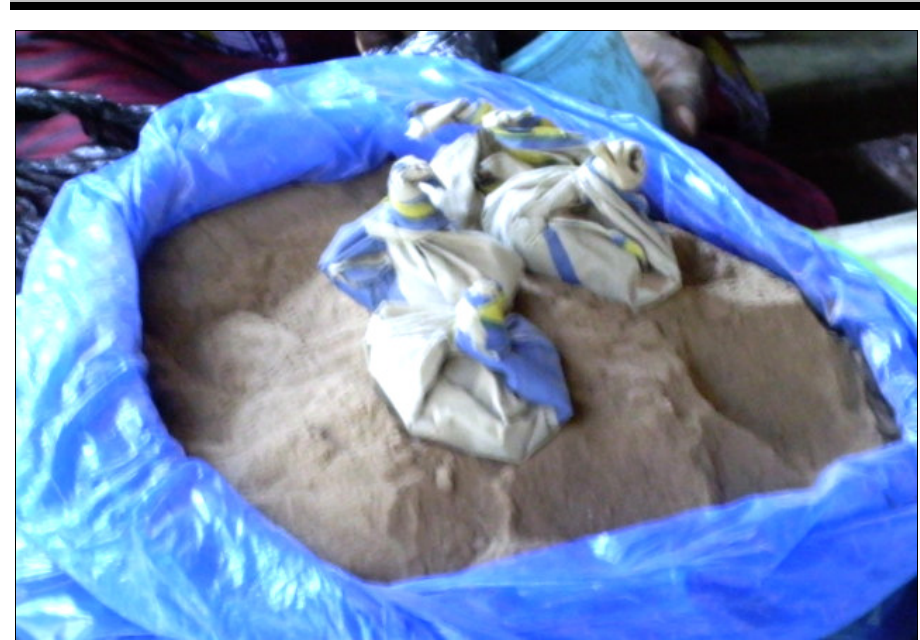

Figure 1: Beilschmiedia mannii (Meisn.) Benth. et Hook. (Lauraceae) : Poudre de graines pilées ou bien pulvérisées

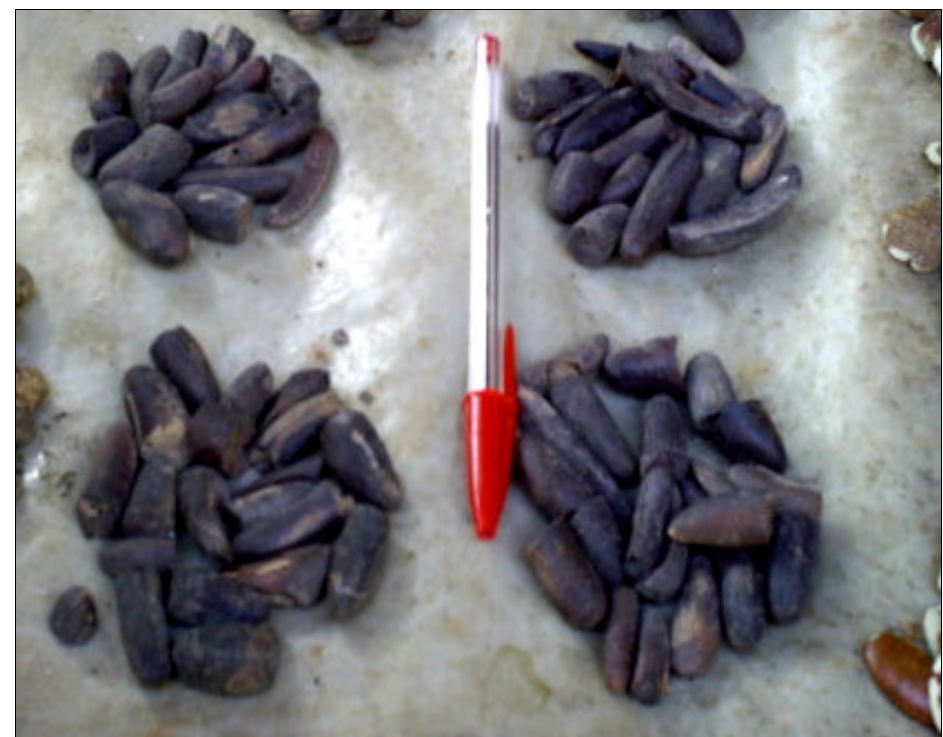

Figure 2 : Beilschmiedia mannii (Meisn.) Benth. et Hook.f. (Lauraceae) : Graines séchées

La connaissance des aliments locaux et leur composition est à la base de certaines des actions conduites pour améliorer la nutrition (évaluation et suivi des apports nutritionnels, planification des ressources alimentaires, réalisation de régimes équilibrés et éducation nutritionnelle). En effet, les plantes spontanées alimentaires se retrouvent de plus en plus sur les marchés et même dans l'alimentation quotidienne des familles, ce qui traduit l'intérêt que les populations portent à ces plantes
(Kouamé, 2000). Toutes ces considérations nous ont permis d'orienter des études approfondies des ressources alimentaires spontanées qui devraient conduire à la mise en évidence des qualités particulières de certaines d'entre elles et contribuer ainsi à leur valorisation. C'est pourquoi une étude physico-chimique, pour la mise en évidence des potentialités nutritionnelles, en occurrence les lipides, les protéines, les sucres et les cendres de sept espèces ont été réalisées. 
MATHERIEL ET METHODE

Matériel d'étude physico-chimique

Matériel biologique : Les organes de végétaux utilisés dans cette étude sont : fruits, graines séchées et feuilles. Parmi les espèces inventoriées, sept d'entre elles, vendues sur les marchés locaux de la Région de Gagnoa situé au Centre-Ouest de la Côte d'Ivoire ont fait l'objet d'une étude monographique et d'analyse physicochimique de leurs organes. Ce sont: Beilschmiedia mannii (graines), Irvingia gabonensis (graines), Myrianthus arboreus (feuilles), Treculia africana (fruits), Ricinodendron heudelotii (graines), Strombosia pustulata (graines) et Sesamum indicum (graines).

\section{Matériel technique}

Méthode de caractéristiques physico-chimiques

Détermination de la teneur en humidité : Les organes ont été prélevés dans trois villages du département de Gagnoa au Centre-Ouest de la Côte d'Ivoire à Djérégoué, Gnagbodougnoa et Bamo. Le prélèvement s'est effectué par intervalle de dix jours, sur un mois, pendant la période de maturité, pour chaque plante. Après les prélèvements, des échantillons de plus de dix kilogrammes sont transportés à la station de recherche de C.N.R.A. de Gagnoa. A la station, les graines sont extraites et séchées à l'air libre afin d'éviter leur pourrissement. C'est après les trois échantillonnages que ces organes sont transportés au Laboratoire des sciences des aliments pour la préparation des échantillons en vue des analyses physique et chimique.
Pour la détermination des paramètres physico-chimiques, plusieurs opérations sont effectuées sur les échantillons à utiliser. Les organes à étudier (feuilles, fruits) sont directement placés à l'étuve à $80^{\circ} \mathrm{C}$ pendant 48 heures selon la méthode de B.I.P.A. (1976). Cette méthode consiste à déterminer la perte de poids provoquée par le maintien de l'échantillon dans les conditions de température et de pression définies. La matière sèche obtenue pour chaque organe est transformée en poudre à l'aide d'un broyeur (Moulinex). Le broyat est tamisé avec un tamis de maille de $2 \mathrm{~mm}$ de diamètre. La poudre obtenue est conditionnée en sachet et conservé dans un endroit sec avant les analyses.

Dosage des lipides : La détermination de la teneur en matières grasses de l'échantillon a été réalisée par extraction au soxhlet (B.I.P.E.A., 1976). Au cours de ce dosage, un gramme ( $1 \mathrm{~g})$ d'échantillon a été introduit dans une cartouche d'extraction (cartouche de Wattman) insérée dans l'ampoule d'extraction. Un ballon à fond rond préalablement pesé ( $P 1$ ) a été rempli au $2 / 3$ avec de l'hexane. Ce ballon a été racolé au reste du système réfrigérant pendant 6 heures où la matière grasse est extraite. Le solvant est récupéré par évaporation. Le ballon contenant la matière grasse a été mis à séchage à $130 \mathrm{C}^{\circ}$ pendant 30 minutes à l'étuve, ensuite il a été refroidi au dessiccateur et pesé pour obtenir le poids (P2).

L'Expression des résultats obtenus par le calcul du pourcentage de la matière grasse est la suivante :

$\% M G=P 2-P 1 \times 100 / P E$

(1)

Avec:

$M G=$ matière grasse

$\mathrm{P} 2=$ poids du ballon + masse de la matière grasse $(\mathrm{g}) \mathrm{sec}$

$\mathrm{P} 1=$ poids du ballon vide $(\mathrm{g})$

$\mathrm{PE}=$ prise d'essai $=$ poids de l'échantillon $(\mathrm{g})$

Détermination des paramètres chimiques de l'huile : Les caractéristiques chimiques (Indice de saponification, Indice de peroxyde, acidité et Indice d'iode) sont déterminées par la méthode volumétrique.

Indice de saponification (Is) : La méthode utilisée est celle du B.I.P.E.A. (1976). Un gramme de matière grasse a été pesé dans un ballon à fond plat auxquels ont été ajoutés $10 \mathrm{ml}$ de potasse alcoolique $0,5 \mathrm{~N}$. L'ensemble adapté à un réfrigérant ascendant a été porté au bainmarie bouillant pendant une heure en agitant de temps en temps. Pour s'assurer que les acides libres contenus dans la matière grasse ont été neutralisés, on y a ajouté $5 \mathrm{ml}$ d'eau, 3 gouttes de phénolphtaléine. L'excès de potasse a été titré avec l'acide chlorhydrique $\left(\mathrm{H}_{2} \mathrm{SO}_{4}\right)$ 0,5 $\mathrm{N}$ jusqu'à la décoloration totale. 


\section{Kouame et al. J. Appl. Biosci. Étude physico-chimique de sept (7) plantes spontanées alimentaires du centre-ouest de la Côte d'Ivoire}

La formule permettant de calculer l'indice de saponification est la suivante:

(2)

Is = (V-Ve) $x$ Nx PM (KOH)/ P

Avec:

* V et $\mathrm{Ve}($ en $\mathrm{ml})=$ volumes de $\mathrm{HCl} 0,5 \mathrm{~N}$ utilisés pour titrer respectivement la potasse dans l'essai à blanc et dans les échantillons de l'huile de plante alimentaires spontanée

${ }^{*} \mathrm{~V}=$ Volume de $\mathrm{Hcl}$ versé dans le blanc (ml)

* $V e=$ Volume de Hcl versé dans l'échantillon (ml)

${ }^{*} \mathrm{~N}=$ Normalité de $\mathrm{Hcl}$

${ }^{*} \mathrm{PM}(\mathrm{KOH})=$ poids moléculaire de la potasse $(\mathrm{g})$

${ }^{*} \mathrm{P}=$ poids de la matière grasse en grammes $(\mathrm{g})$

Indice de peroxyde (Ip) : La méthode utilisée est celle de COCKS. Cette méthode à été mise en place en 1966. (c'est avec cette méthode que j'ai pu faire l'analyse ) Un

L'expression de l'indice de

(3)

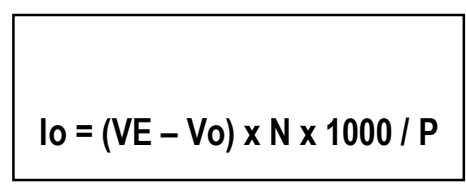

gramme de matière grasse a été pesé dans un Erlenmeyer de $250 \mathrm{ml}$ auquel ont été ajoutés $25 \mathrm{ml}$ de solvant d'acide acétique et de $25 \mathrm{ml}$ chloroforme. L'agitation est faite de manière régulière jusqu'à ce que la matière grasse soit dissoute dans le solvant. Le mélange a été laissé au repos pendant 15 minutes avec une agitation d'une minute par intervalles de 5 minutes, ensuite il y a un ajout de $35 \mathrm{ml}$ d'eau distillée, 3 gouttes d'empois d'amidon $1 \%$. Enfin ce mélange a été dosé avec le thiosulfate de sodium $\left(\mathrm{Na}_{2} \mathrm{~S}_{2} \mathrm{O}_{3}\right) 0,01 \mathrm{~N}$ jusqu'à la disparition de la couleur jaunâtre.

peroxyde est la suivante :
Avec:

$V_{0}=$ Volume de Thiosulfate $\left(\mathrm{Na}_{2} \mathrm{~S}_{2} \mathrm{O}_{3}\right)$ versé dans le blanc (ml)

VE = Volume de Thiosulfate $\left(\mathrm{Na}_{2} \mathrm{~S}_{2} \mathrm{O}_{3}\right)$ versé dans l'échantillon (ml)

$\mathrm{N}=$ Normalité de Thiosulfate $\left(\mathrm{Na}_{2} \mathrm{~S}_{2} \mathrm{O}_{3}\right)$

$\mathrm{P}=$ prise d'essai $(\mathrm{g})$

Dosage de l'acidité : Il s'agit de mesurer l'acidité titrable avec une solution titrante d'hydroxyde de sodium $(0,1 \mathrm{~N})$ en présence d'un indicateur de fin de réaction (phénolphtaléine). La méthode utilisée est celle de (B.I.P.E.A, 1976). Un gramme de matière grasse a été pesé dans un Erlenmeyer de $250 \mathrm{ml}$ auquel ont été ajoutés $25 \mathrm{ml}$ de chloroforme et le tout a été laissé au repos jusqu'à la dispersion de la matière grasse. Vingt cinq d'éthanol $95 \%$ et 8 gouttes de phénolphtaléine (1 $\%$.) sont ajoutés. Le dosage se fait avec la soude alcoolique ( $\mathrm{NaOH} 0,05 \mathrm{~N})$. Le pourcentage de l'indice d'acidité est calculé de la façon suivante :
(4)

$\%$ AGL $=($ Vo-VE) $\times \mathrm{N} \times 28,2 / \mathrm{P}$

(5)
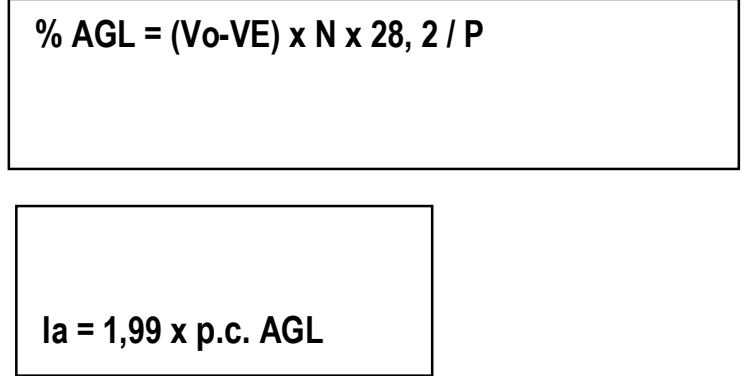
Avec:

$V_{0}=$ Volume de $\mathrm{NaOH}$ versé dans le blanc $(\mathrm{ml})$

$\mathrm{VE}=$ Volume de $\mathrm{NaOH}$ versé dans l'échantillon (ml)

$\mathrm{N}=$ Normalité de la soude

$\mathrm{P}=$ poids de l'échantillon $(\mathrm{g})$

Indice d'iode : La méthode utilisée est celle de WOLF.

Un gramme de matières grasses dans un flacon de 300 à

$500 \mathrm{ml}$ a été dissout avec $15 \mathrm{ml}$ de tétrachlorure de

carbone $\left(\mathrm{CCl}_{4}\right)$ et $25 \mathrm{ml}$ de réactif de WISSON ont été ajoutés. Les flacons bouchés ont été agités légèrement puis ont été placés à l'obscurité pendant 2 heures. On ajoute $20 \mathrm{ml}$ de $\mathrm{KI}$ (lodure de potassium) à $10 \%$ et 150 $\mathrm{ml}$ d'eau distillée. Le dosage est fait avec l'oxyde de sulfate de soude $\left(\mathrm{Na}_{2} \mathrm{~S}_{2} \mathrm{O}_{3}\right) 0,1 \mathrm{~N}$ en présence d'empois d'amidon jusqu'à la disparition totale de la coloration rouge. Un essai à blanc a été fait dans les mêmes conditions que l'essai pour titrer le $\mathrm{CCl}_{4}$.

L'expression de l'indice d'iode est la suivante:

(6)

$$
\mathrm{li}=127 \mathrm{X}(\mathrm{V}-\mathrm{Ve}) \times \mathrm{N} / 10 \times \mathrm{P}
$$

Avec:

$\mathrm{P}=$ le poids de la matière grasse en grammes

$v$ et ve sont les volumes de thiosulfate de sodium

versés respectivement pour le blanc et pour l'échantillon (ml)

$V e=$ Volume de $\mathrm{Na}_{2} \mathrm{~S}_{2} \mathrm{O}_{3}$ versé dans l'échantillon $(\mathrm{ml})$

$\mathrm{V}=$ Volume de $\mathrm{Na}_{2} \mathrm{~S}_{2} \mathrm{O}_{3}$ versé dans le blanc $(\mathrm{ml})$

$\mathrm{N}=$ normalité de $\mathrm{Na}_{2} \mathrm{~S}_{2} \mathrm{O}_{3}$

127 = poids atomique de l'iode

Dosage des Protéines : La méthode utilisée pour la détermination des protéines est celle de KJELDAHL

(B.I.P.E.A.,

1976). Cette méthode comprend deux grandes étapes essentielles :

- $\quad$ La minéralisation sulfurique (Digestion) ;

- $\quad$ La distillation suivie du titrage avec l'acide chlorhydrique $(\mathrm{HCl})$.

Minéralisation sulfurique (digestion): Un gramme d'échantillon a été introduit dans des tubes matras de KJELDAHL (B.I.P.E.A., 1976). Dans ces tubes, ont été ajoutés $12 \mathrm{ml}$ d'acide sulfurique $\left(\mathrm{H}_{2} \mathrm{SO}_{4}\right)$ à $98 \%$ et une pincée de pastilles de sulfate de cuivre $\left(\mathrm{CuSO}_{4}\right)$ a été utilisée comme catalyseur. Le sulfate de potassium $\left(\mathrm{K}_{2} \mathrm{SO}_{4}\right)$ a été ajouté pour élever le point d'ébullition de $\mathrm{H}_{2} \mathrm{SO}_{4}$. Apparition d'une fumée blanche qui montre que l'évaporation de l'eau est achevée et que la liqueur obtenue a une coloration vert clair. C'est le sulfate d'ammonium.

Distillation suivie du titrage avec l'acide chlorhydrique : La distillation est la décomposition du Sulfate d'ammonium par la soude $(0.5 \mathrm{~N})$, l'ammoniac libéré est entraîné par la vapeur d'eau. Cet ammoniac a été titré à l'aide d'une burette contenant de l'acide chlorhydrique $(0.109 \mathrm{~N})$ en présence d'un indicateur coloré qui est le rouge de méthyle. Le titrage est achevé lors du virage du bleu au rouge. Le résultat obtenu, après titrage, nous permet de calculer le pourcentage d'azote total.

La formule est la suivante :

$$
\% \text { Azoté total }=[\mathrm{VHCl}-\mathrm{VHCl} \times \mathrm{NHCl} X 1.401] / \mathrm{P}
$$

Avec:

$\mathrm{VHCl}=$ Volume de l'acide chlorhydrique pour l'échantillon

(ml)

$\mathrm{NHCl}=$ Normalité de l'acide chlorhydrique $=0,9516$

$V_{0}=$ Volume de l'acide chlorhydrique versé dans le blanc

(ml)

Facteur de conversion $=6,25$
Constante $=1,401$

Prise d'essai $=1$ gramme

Pourcentage de protéines $=$ pourcentage d'Azote total $\mathrm{x}$ 6,25

Dosage quantitatif des cendres ( $\mathrm{Ca}, \mathrm{Mg}, \mathrm{K}, \mathrm{Na}$ et $\mathrm{P})$ : Les cendres ont été déterminées selon la méthode A.O.A.C (1975). Une quantité de un gramme de chaque 


\section{Kouame et al. J. Appl. Biosci. Étude physico-chimique de sept (7) plantes spontanées alimentaires du centre-ouest de la Côte d'Ivoire}

échantillon a été prélevée et mise dans le creuset préalablement taré. La masse de l'ensemble creuset et échantillon est notée Mce. Cet ensemble a été placé dans le four à moufle à $550^{\circ} \mathrm{C}$ pendant 48 heures. Au bout du temps d'incinération, l'ensemble est retiré du four et déposé dans un dessiccateur pour le refroidissement ; ensuite les pesés sont effectuées.

Le taux de cendre est déterminé par la formule suivante :

(8)

$$
\% \text { Cendre }=\text { Mcc }- \text { Mcv } \times 100 / \text { Mce-Mcv }
$$

Avec :

$\mathrm{Mcc}=$ Masse de creuset + cendre $(\mathrm{g})$

$\mathrm{Mcv}=$ Masse de creuset vide (g)

Mce $=$ Masse de creuset + échantillon $(\mathrm{g})$

Dosage des sucres libres ou solubles: L'extraction des sucres solubles est faite selon la méthode de Dubois et al. (1956) et modifié par Agbo et al. (1986). Elle comprend une phase d'extraction proprement dite et une phase de défécation (purification en sucres). La quantité prélevée est fixée à l'alcool bouillant suivant la figure 14 . Phase d'extraction : La phase d'extraction est illustrée par la figure 14. Pour réaliser cette phase, deux grammes d'échantillon de chaque plante sont pesés dans les tubes à essai auxquels sont ajoutés $10 \mathrm{ml}$ d'éthanol Gay-
Lussac. Le chauffage se fait au bain-marie bouillant à $95^{\circ}$ sous agitation, toutes les 3 minutes. La solution alcoolique (1) obtenue est bien mélangée et la centrifugation est faite à 3000 tours/min pendant $3 \mathrm{~min}$. Le surnageant (S1) a été prélevé, le culot (C1) récupéré auquel sont ajoutés $5 \mathrm{ml}$ d'éthanol suivi d'un chauffage au bain-marie bouillant à $85^{\circ} \mathrm{C}$ pendant $15 \mathrm{~min}$. La solution alcoolique (2) est mélangée à nouveau et la centrifugation est faite comme précédemment. Le surnageant (S2) est prélevé et le culot (C2) est gardé. L'expérience est répétée comme la première fois, mais avec $10 \mathrm{ml}$ d'éthanol. Enfin le culot (C3) est abandonné et le surnageant (S3) a été utilisé pour le dosage des sucres. 


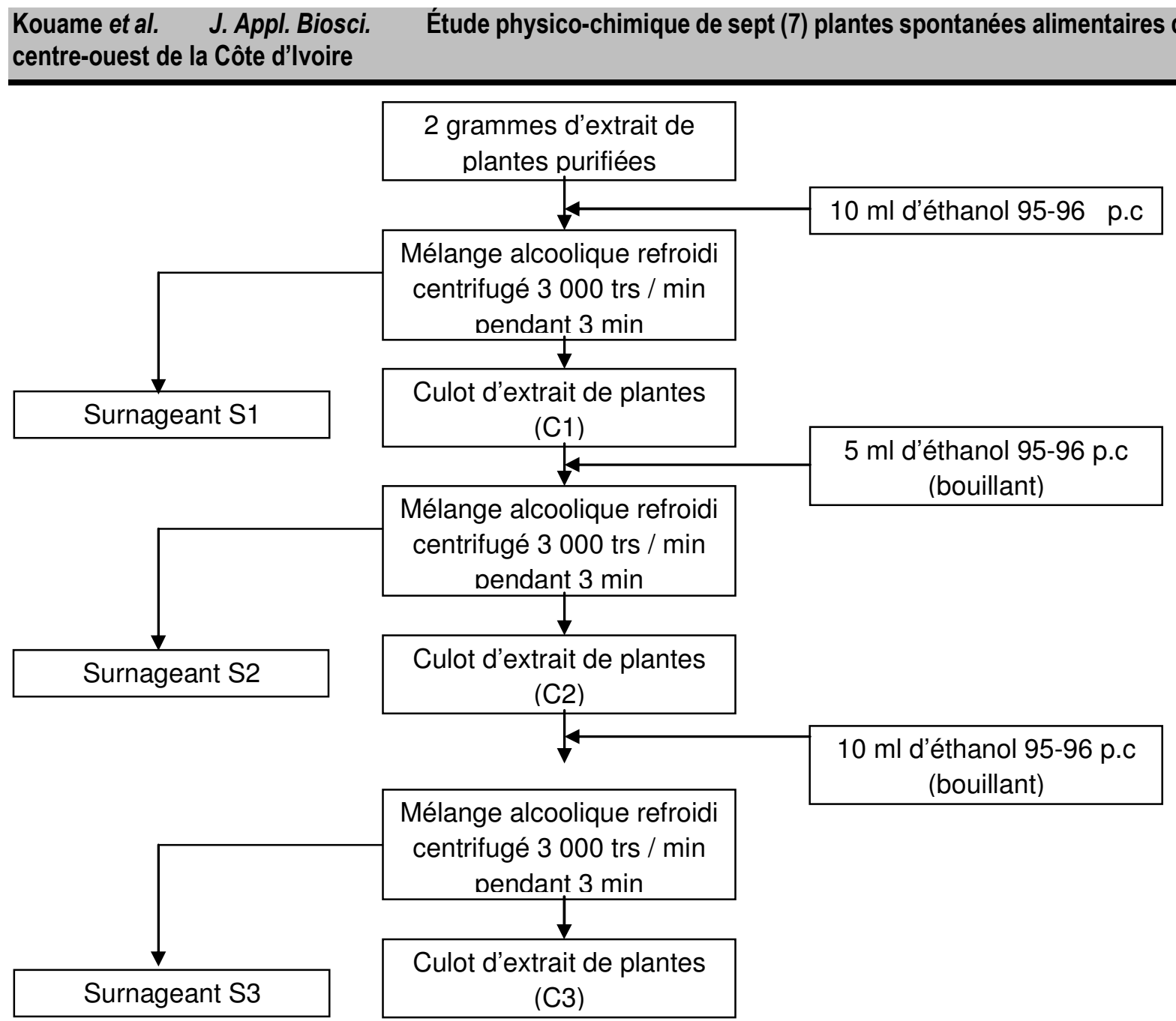

Figure 3 : Schéma du mode d'extraction des sucres libres à partir des extraits des organes des plantes alimentaires spontanées

Phase de défécation: Au niveau de la phase de défécation, les surnageant $\mathrm{S1}, \mathrm{S} 2$, et $\mathrm{S} 3$ sont mélangés dans un tube $(30 \mathrm{ml})$ auquel ont été ajoutés $2 \mathrm{ml}$ d'acétate de plomb (10\%.) et la centrifugation est faite à 3000 tours/min, pendant 3 minutes. Le culot C4 obtenu est rejeté. Le surnageant $\mathrm{S} 4$ est additionné $2 \mathrm{ml}$ d'acide oxalique $(10 \%$.). La centrifugation est faite à 3000 tours/min, pendant 3 minutes comme précédemment etle surnageant (S5) a été versé dans une fiole de $25 \mathrm{ml}$ et placé au bain de sable jusqu'à évaporation de l'excès d'éthanol. Le contenu de la fiole est complété avec de l'eau distillée jusqu'au trait de jauge. Le mélange obtenu sert de solution-mère pour des dosages de sucres réducteurs et totaux. Cette phase est représentée par la figure 15.
Détermination quantitative des sucres solubles (sucres réducteurs) : Pour préparer la solution de DNS, il faut peser $2 \mathrm{~g}$ de DNS dans $40 \mathrm{ml}$ d'eau et puis $3,2 \mathrm{~g}$ de soude $(\mathrm{NaOH})$ pastille qui sont dissous dans $30 \mathrm{ml}$ d'eau. Après le mélange des 2 solutions, sont ajoutés lentement, sous agitation chauffante, $60 \mathrm{mg}$ de tartrate double de solution de potassium $(\mathrm{Na}, \mathrm{K})$ puis nous complétons à $200 \mathrm{ml}$ avec de l'eau distillée. Les tubes sont portés au bain-marie à $100{ }^{\circ} \mathrm{C}$, pendant 5 minutes. Après refroidissement, $20 \mathrm{ml}$ de l'eau distillée sont ajoutés à chaque tube. La lecture se fait au spectrophotomètre à $540 \mathrm{~nm}$. Les densités optiques (D.O.) obtenues sont utilisées pour déduire la concentration en sucres réducteurs en se servant de la courbe d'étalonnage déjà établie. 


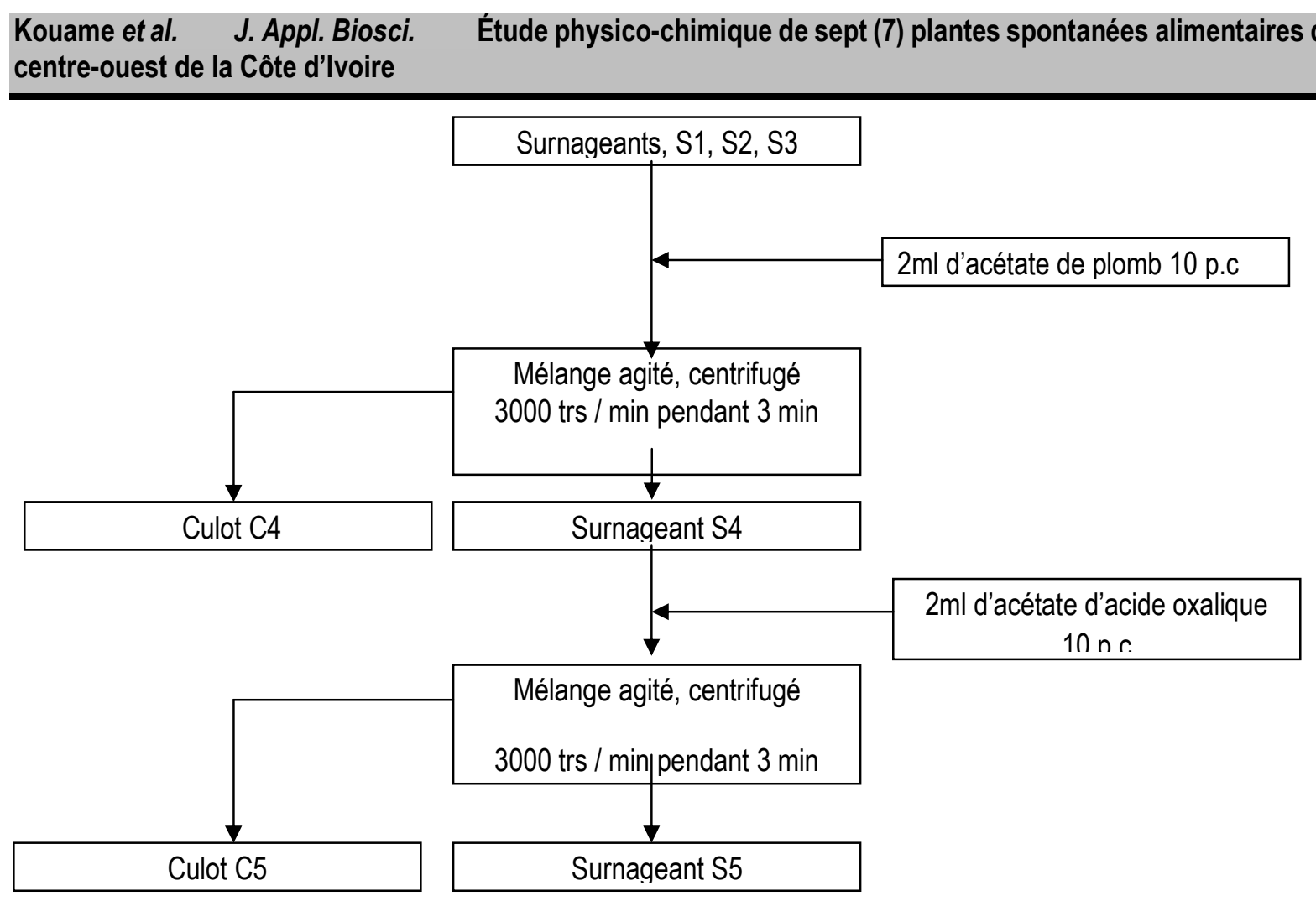

Figure 4 : Schéma du mode de défécation des extraits des organes de plantes alimentaires spontanées

Dosage des sucres totaux par la méthode aux phénols sulfuriques : Les sucres totaux, en présence de l'acide sulfurique concentré se déshydratent pour former du furfural et des dérivés qui, en présence de phénol, donnent un complexe coloré qui est mesuré au spectrophotomètre à $490 \mathrm{~nm}$. Les valeurs se rapportant au contenu des tubes sont lues au spectrophotomètre après refroidissement. Chaque essai est réalisé trois fois. Pour le dosage des sucres totaux, des dilutions

\section{RESULTAT}

L'analyse comparative des nutriments des plantes spontanées alimentaires a porté essentiellement sur les organes consommés :

- des graines de Beilschmiedia mannii, Irvingia gabonensis, Ricinodendron heudelotii, Sesamum indicum, Strombosia pustulata ;

- des feuilles de Myrianthus arboreus ;

- des fruits de Treculia africana.

Les résultats des analyses de la composition physicochimique des graines permettent de les classer en deux catégories :

- $\quad$ graines oléagineuses (teneur en lipide variant de 59 à $84 \%$.) avec Irvingia gabonensis, Ricinodendron heudelotii et Sesamum indicum ; d'échantillons au 1/10ème sont réalisés pour des commodités de lecture. Les résultats obtenus sont des densités optiques (D.O.). Ces D.O. sont utilisées pour déduire la concentration en sucres totaux en se servant de la courbe d'étalonnage. Les différentes concentrations en sucres totaux ainsi obtenues sont utilisées pour calculer la teneur de sucres totaux dans $2 \mathrm{~g}$ d'échantillon de plante.

- graines protéagineuses (teneur en protéine variant de 43 à $53 \%$.) avec Beilschmiedia mannii et Strombosia pustulata.

\section{Graines oléifères}

Teneurs moyennes en lipides de quelques taxons: Les résultats du Tableau 1 montrent que les graines de Irvingia gabonensis ont la plus forte teneur en huile $(83,51 \%$.), par rapport à celles des graines de Sesamum indicum $(65,26 \%$.) et de Ricinodendron heudelotii $(58,76$ $\%$.). La différence de teneur en huile est significative entre les organes des trois espèces. Les graines de Strombosia pustulata $(19,9 \%$.) et de Beilschmiedia mannii $(13,09 \%$.) ont de faibles teneurs en huile. Les graines de Ricinodendron heudelotii et de Sesamum indicum se distinguent des graines de Irvingia gabonensis 


\section{Kouame et al. J. Appl. Biosci. Étude physico-chimique de sept (7) plantes spontanées alimentaires du centre-ouest de la Côte d'Ivoire}

par leur taux élevés en protéine (33 et $23 \%$.) et en cendres $(9,4$ et 7,5$)$. Mais les graines de Ricinodendron heudelotii révèlent une teneur très pauvre en sucre $(0,8$ $\%$.).
Paramètres chimiques des huiles : Les résultats des analyses des indices d'acidité (la), d'iode (lo), de saponification (IS) et de peroxyde (Ip) des oléagineuses sont consignés dans le tableau 2.

Tableau 1 : Composition physico-chimique des organes des espèces végétales

\begin{tabular}{|l|l|l|l|l|l|l|}
\hline \multicolumn{2}{|c|}{} & $\begin{array}{c}\text { Lipides } \\
(\% \% \text { matière } \\
\text { sèche) }\end{array}$ & $\begin{array}{c}\text { Protéines } \\
(\% \text { matière } \\
\text { sèche) }\end{array}$ & $\begin{array}{c}\text { Cendres } \\
\text { (\%matière } \\
\text { sèche) }\end{array}$ & $\begin{array}{c}\text { sucres } \\
(\% \text { matière } \\
\text { sèche) }\end{array}$ & $\begin{array}{c}\text { \%de } \\
\text { matière } \\
\text { sèche }\end{array}$ \\
\hline \multirow{2}{*}{$\begin{array}{l}\text { Graines } \\
\text { oléagineuses }\end{array}$} & Irvingia gabonensis & $\mathbf{8 3 , 5 1}$ & 7,39 & 2,9 & $\mathbf{5 , 5 8}$ & 21,62 \\
\cline { 2 - 7 } & Ricinodendron heudelotii & $\mathbf{5 8 , 7 6}$ & 33 & 7,5 & $\mathbf{0 , 8}$ & 24,9 \\
\cline { 2 - 8 } & Sesamum indicum & $\mathbf{6 5 , 2 6}$ & 23,27 & 9,4 & $\mathbf{2 , 0 5}$ & 21,24 \\
\hline \multirow{2}{*}{$\begin{array}{l}\text { Graines } \\
\text { Protéagineuses }\end{array}$} & Beilschmiedia mannii & 13,09 & $\mathbf{4 2 , 8 5}$ & $\mathbf{3 6 , 6 8}$ & $\mathbf{7 , 4 4}$ & 15,58 \\
\cline { 2 - 8 } & Strombosia pustulata & 19,90 & $\mathbf{5 3 , 0 3}$ & 15 & $\mathbf{8 , 7}$ & 17,8 \\
\hline Feuilles & Myrianthus arboreus & 5,8 & $\mathbf{5 7 , 0 2}$ & $\mathbf{3 6}$ & $\mathbf{1 , 0 4}$ & $\mathbf{3 0 , 6 9}$ \\
\hline Fruits & Treculia africana & 16 & $\mathbf{3 3 , 8}$ & $\mathbf{2 7}$ & $\mathbf{2 3 , 1 2}$ & 17,3 \\
\hline
\end{tabular}

Tableau 2 : Paramètres chimiques des huiles contenues dans les graines de Irvingia gabonensis, de Ricinodendron heudelotii et de Sesamum indicum

\begin{tabular}{|l|c|c|c|c|}
\hline \multicolumn{1}{|c|}{ Différents indices } & $\begin{array}{c}\text { Indice } \\
\text { d'Acidité }\end{array}$ & $\begin{array}{c}\text { Indice } \\
\text { D'lode }\end{array}$ & $\begin{array}{c}\text { Indice de } \\
\text { Saponification }\end{array}$ & $\begin{array}{c}\text { Indice de } \\
\text { Peroxyde }\end{array}$ \\
\hline Irvingia gabonensis & 6,77 & $\mathbf{1 3 4 , 7 6}$ & $\mathbf{1 7 8 , 7 5}$ & 3,22 \\
\hline Ricinodendron heudelotii & $\mathbf{1 2 , 2 9}$ & 106,03 & 169,50 & $\mathbf{2 3 , 7 4}$ \\
\hline Sesamum indicum & 12,19 & 65,70 & 134,42 & 13,74 \\
\hline
\end{tabular}

Indices de peroxyde (Ip): Toutes les huiles des organes des plantes ont montré des différences significatives au cours de l'analyse statistique. Quant aux indices de peroxyde. L'huile de Ricinodenddron heudelotii a la valeur la plus élevée en Ip $(23,87 \pm 0,12$.). Par rapport à celle de l'huile de Irvingia gabonensis avec une valeur moyenne de $(13,74 \pm 0,12)$ L'huile de Sesamum indicum présente l'Ip le plus faible $(3,22)$.

Indices d'acidité (la): La différence est significative entre les valeurs de l'indice d'acidité des huiles des graines des plantes analysées. L'huile de Irvingia gabonensis, a une acidité qui est de 6,77, celle de Ricinodendron heudelotii et de Sesamum indicum ont des indices d'acidités respectives de 12,29 et de 12,19.

Indices d'iode (I0): Les huiles des graines des plantes analysées présentent une différence significative pour les indices d'iode. L'huile de l'amande de Irvingia gabonensis a un indice d'iode plus élevé $(134,76 \pm 0,84)$. Par rapport à celle du Ricinodendron heudelotii $(106,02 \pm 0,84 \%$.) L'indice d'iode le plus faible est celle de Sesamum indicum avec une valeur de 65,69.
Graines protéagineuses :Les graines protéagineuses sont des graines qui ont une forte teneur en protéines. Au terme des analyses, les résultats révèlent que les graines de Strombosia pustulata et Beilschmiedia mannii renferment chacune une teneur en protéine respective de 53,03 et $42,85 \%$ Cette teneur est plus élevée dans les graines de Strombosia pustulata (53\%). Les résultats du tableau $\mathrm{V}$ indiquent que les graines protéagineuses se distinguent des graines oléagineuses par leurs teneurs élevées en cendres et en sucres. Ce sont : Beilschmiedia mannii avec $36,68 \%$ en cendres, et 7,44 \% en sucres et Strombosia pustulata avec $15 \%$ en cendres et $8,7 \%$ en sucres. Les graines de Ricinodendron heudelotii et de Sesamum indicum (oléagineuses) présentent aussi des teneurs importantes en protéine, avec respectivement 33 et $23 \%$ Ces teneurs en protéines restent inférieures à celles de Beilschmiedia mannii et de Strombosia pustulata. Les teneurs en sucres les plus faibles sont observées dans les graines de Ricinodendron heudelotii $(0,8 \%$.) et de Sesamum indicum $(2,05 \%$.). La différence 


\section{Kouame et al. J. Appl. Biosci. Étude physico-chimique de sept (7) plantes spontanées alimentaires du centre-ouest de la Côte d'Ivoire}

est significative pour les teneurs en cendres et en sucres des graines oléagineuses et des graines protéagineuses.

Feuilles et fruits : Les résultats du tableau $\mathrm{V}$ montrent que les feuilles de Myrianthus arboreus présentent des teneurs élevées en protéines et en cendres, soit respectivement $57,02 \%$ et $36 \%$., tandis que les taux les

\section{DISCUSSION}

\section{Graines oléifères}

Teneurs moyennes en lipides de quelques taxons: L'évaluation de la teneur en huile a montré que les graines de Irvingia gabonensis, de Sesamum indicum et de Ricinodendron heudelotii sont riches en huiles. Leurs teneurs respectives sont : $83,51 \%$., $65,26 \%$ et $58,76 \%$ Ces analyses ont montrées que les graines sèches de Irvingia gabonensis ont une teneur en huile supérieure à celle obtenue par Kouakou (2002) qui est de $70 \%$., par Silou et al. (2004) qui est de $60 \%$ et par Traoré (2005) qui est de $73 \%$ Cette teneur en huile se situe au-dessus de la limite indiquée par Joseph (1995) qui varient de 71 $\%$ à $76 \%$ Cette différence de résultats est due à la méthode d'extraction, mais aussi à la provenance des graines car les variations saisonnières influencent les résultats de la composition physico-chimique des organes des plantes. Les graines de Irvingia gabonensis ont une teneur en huile qui est largement supérieure à celle des graines fraîches de palmier à huile (45 à $50 \%$.), de soja (18\%.), de coton (18\%.), de tournesol (18\%.) et de l'arachide (46\%.). Selon Silou et al. (2004), les graines de Irvingia gabonensis ont une huile alimentaire qui peut avoir des utilisations industrielles, en margarinerie, en savonnerie et en cosmétique. C'est pourquoi, l'on doit se pencher sur la valorisation de ces plantes spontanées. La teneur en huile des graines de Ricinodendron heudelotii (59\%.) est supérieure à celle obtenue par Tapi (2003) pour la même espèce (48\%). Cette teneur en huile de Ricinodendron heudelotii est supérieure à celle de l'arachide (46\%.), de palmier à huile (45\% à $50 \%$.), de soja, de coton et de tournesol, des graines de Ricinodendron heudelotii obtenue par Busson (1965) qui est de $44 \%$ Voyant, la teneur en huile de Ricinodendron heudelotii. La teneur en huile des graines de Sesamum indicum est inférieure à celle de Irvingia gabonensis et supérieure à celle de Ricinodendron heudelotii. Cette teneur est par contre supérieure à celle des graines fraîches de palmier à huile et des graines d'arachide. Les graines de Ricinodendron heudelotii présentent également une teneur en sucres très faible $(0,8 \%$.) par rapport à celle des graines de Sesamum indicum $(2,05$ $\%$.). La consommation de ces graines oléagineuses peut être conseillée aux diabétiques. Comparer aux aliments plus faibles s'observent en teneurs en sucre $(1,04 \%$.) et en lipide $(5,8 \%)$. Pour les fruits de Treculia africana, toutes les teneurs restent élevées. La plus élevée est la teneur en protéines avec 33,8 \%., après viennent les teneurs en cendres $(27 \%$.), en sucres $(23,12 \%$.) et enfin la teneur en lipides $(16 \%$.).

usuels riches en éléments nutritifs, les espèces spontanées consommées se révèlent quelques fois plus riches et plus onéreux ce qui nécessite leurs valorisation pour les besoins nutritionnelles des populations. Concernant la valorisation et l'utilisation en agroalimentaire industriel possible de ces ressources locales, une étude supplémentaire de la caractérisation des propriétés physico-chimiques des huiles a été effectuée, c'est la détermination des différents indices.

Paramètres chimiques des huiles : L'indice d'iode de l'huile de Irvingia gabonensis $(134,76)$ est largement supérieur ceux des huiles de Ricinodendron heudelotii $(106,03)$, de Sesamum indicum $(65,70)$ et de Irvingia gabonensis obtenu par Traoré (2005). Ces indices sont supérieurs à ceux de l'huile d'arachide (84 à 100), du beurre de karité (52 à 66), de l'huile de palme (45 à 58) et de l'huile de coton (99 à 115). Cependant, les valeurs des li de Irvingia gabonensis et de Ricinodendron heudelotii sont sensiblement égales à celles du tournesol (125 à 136) et du soja (125 à 138). Par conséquent, ces valeurs restent très élevées et montrent que ces huiles sont insaturées. On peut donc dire qu'elles sont riches en acides gras essentiels, importantes pour la consommation. L'indice d'iode de l'huile de Ricinodendron heudelotii $(106,03$.) est identique à celui des cotons (99 à 115) et supérieurs à celui de l'arachide (84 à 100). Compte tenu du fait que le coton et l'arachide possède de très bonnes huiles consommables, on peut conclure que l'huile de Ricinodendron heudelotii est de la bonne qualité et consommable. Mais cette huile reste cependant un peu plus acide. Son indice d'acidité (la) est $12,29 \%$ La valeur de 169,50 \% d'indice de saponification est inférieure à la valeur obtenue par Tchiegang et al. (2005) qui est de 193,73 à 195,02 \% Cette différence peut être due à l'origine de l'espèce ou à la méthode d'extraction et aux traitements appliqués aux amandes. Tchiegang et al. (2005) ont signalé la qualité et la stabilité de l'huile pendant 4 mois de conservation. L'huile de Sesamum indicum a un indice de saponification de 134,43 . Cet indice est inférieur à celui déterminé par Buson (1965) qui de 191. Cette différence peut être due à l'origine de l'échantillon. En considérant les résultats de Busson (1965), on peut estimer l'Is de Sesamum indicum 


\section{Kouame et al. J. Appl. Biosci. Étude physico-chimique de sept (7) plantes spontanées alimentaires du centre-ouest de la Côte d'Ivoire}

entre 134 et 191. Ces valeurs sont dans le même ordre que celles des indices de saponification du beurre de karité, des huiles de soja (188 à 195), de coton (190 à 198), de l'arachide (189 à 196) et du tournesol (188 à 193). Cette huile a une qualité qui est similaire à celle des huiles précitées. Selon Busson (1965), Sesamum indicum est riche en acide palmitique $(9,8)$, acide stéarique $(6,8)$, acide oléique $(49,2)$ et en acide linoléique $(34,2)$. L'huile de sésame est très employée comme huile de table ou pour la fabrication de margarines. L'huile des graines de Irvingia gabonensis a un indice de saponification $(178,75)$ supérieur à ceux des graines de Ricinodendron heudelotii $(169,50)$ et de Sesamum indicum $(134,42)$. La comparaison de l'indice de saponification de Irvingia gabonensis à ceux de certains végétaux montre qu'il est sensiblement égal à celui de beurre de karité compris entre 178 et 193. Ces comparaisons permettent de dire que l'huile de Irvingia gabonensis sont servir dans la fabrication des produits cosmétiques parce qu'elle donne un indice d'acidité nul. L'indice de peroxyde de l'huile des amandes de Irvingia gabonensis est faible $(3,22 \%$.). Cela montre que cette huile ne s'oxyde pas facilement, elle peut être conservée longtemps. Les beurres et graisses qui, dans les conditions normales sont solides, ont un indice d'iode inférieur ou égal à 70 , ce qui est le cas de Sesamum indicum (65,69\%.). En outre, le savon fabriqué à partir d'un corps gras à indice d'iode élevé a tendance à être mou. Pour le cas de Irvingia gabonensis, l'indice d'iode étant élevé et la graisse solide, les savons formés peuvent l'être aussi. L'huile végétale joue un rôle de plus en plus important dans l'approvisionnement global en matières grasses. Selon Busson (1965), avant le XVème siècle, les huiles végétales constituaient la principale source lipidique de l'alimentation humaine en Afrique de l'Ouest. Elles sont utilisées soit dans l'industrie alimentaire ou dans les domaines pharmaceutique et technique, soit dans les deux cas. On peut citer les huiles de soja, du tournesol, de coton, du maïs et de l'olive à $100 \%$ pour la consommation humaine. Les huiles de lin et de ricin sont exclusivement réservées à l'industrie non alimentaire. Les graines de Irvingia gabonensis, de Ricinodendron heudelotii et de Sesamum indicum ont des huiles de qualité similaire à celle des huiles des espèces précitées. Les huiles des espèces étudiées peuvent être utilisées soit dans l'industrie alimentaire, soit dans le domaine pharmaceutique et technique.

Graines protéagineuses : Les graines de Beilschmiedia mannii et de Strombosia pustulata ont une quantité importante de protéines variant entre 43 et $53 \%$. Mais ces teneurs en protéines restent très élevées par rapport à celles déterminées par Busson (1965) avec des graines des espèces en provenance de Duékoué $(12,52 \%$.). Les graines de Ricinodendron heudelotii ont un taux de protéines de $33 \%$ supérieur au taux de protéines de l'arachide $(23,2 \%$.), de niébé $(23,1 \%$.) et de haricot $(21,7 \%$.), mais sensiblement égale au taux de protéines contenu dans les graines du Soja $(33,7 \%$.). Ces analyses permettent de classer Ricinodendron heudelotii comme une oléagineuse parmi les protéagineuses. Les graines de Beilschmiedia mannii et de Strombosia pustulata présentent des teneurs en protéines supérieures à celles de toutes les espèces précitées. Mais les graines de ces deux plantes ont des teneurs en protéines sensiblement égales à celle de Néré (33 à 48 $\%$.). La comparaison des protéines des graines oléagineuses montre que les teneurs en protéines de maïs jaune et clair qui varient entre 8,6 et $9,2 \%$ sont légèrement supérieures à la teneur en protéines des graines Irvingia gabonensis (7,4\%.). Les graines de Sesamum indicum ont un taux de protéines de $23,3 \%$ sensiblement égal à celui des graines de l'arachide $(26,4$ à $27,5 \%$.). Les graines de Beilschmiedia mannii se distinguent des graines des autres espèces par une teneur élevée en cendre et sels minéraux (36) \%. II ressort des résultats obtenus que les besoins nutritionnels en protéines et en sels minéraux de la population peuvent être comblés par la consommation des organes de Beilschmiedia mannii, Strombosia pustulata et de Sesamum indicum. II est donc important de faire connaître ces espèces.

Feuilles et fruits: Les analyses ont montré que les feuilles de Myrianthus arboreus contiennent des teneurs très élevées en protéines $(57,02 \%$.) et en cendres (36 $\%$.), mais très pauvres en sucre $(1,04)$ avec peu de lipides $(5,8 \%$.). Ces valeurs nutritives varient dans des proportions aussi importantes que certaines légumes feuilles citées par N'dri (1986). Ce sont : Solanum nigrum (32\% de protéines et $15 \%$ de sels minéraux), Ceiba pentandra $(12,4 \%$ de protéines et $14,4 \%$ de sels minéraux) et Triplochiton scleroxylon (13\% de protéines et $13,8 \%$ de sels minéraux). Ces données permettent de conclure que les feuilles des plantes spontanées alimentaires consommées contribuent à l'équilibre nutritionnel des populations et exercent par conséquent, une influence sur leur santé. Les feuilles de Myrianthus arboreus ont une teneur très faible en sucres conseillé aux diabétiques. Avec tous ces atouts, l'espèce peut faire partie des plantes à promouvoir dans la consommation. Les fruits de Treculia africana renferment $34 \%$ de protéines, $27 \%$ de cendres et de sels minéraux, $23,12 \%$ de sucres et $16 \%$ de lipides. Ces valeurs nutritives varient dans des proportions importantes. La teneur en 


\section{Kouame et al. J. Appl. Biosci. Étude physico-chimique de sept (7) plantes spontanées alimentaires du centre-ouest de la Côte d'Ivoire}

protéines des mésocarpes de Treculia africana analysées dans l'étude est supérieure à celle signalée par N'dri (1986) qui est $16,4 \%$.. La période et le lieu de récolte semblent être des facteurs influents de ces résultats. Les fruits de cette plante rentrent dans la préparation des

\section{CONCLUSION}

Au terme de l'analyse comparative des nutriments, les graines (Beilschmiedia mannii, Irvingia gabonensis, Ricinodendron heudelotii, Sesamum indicum, Strombosia pustulata) se subdivisent en deux groupes. Ce sont :

\section{* Les oléagineuses riches en lipide \\ * Les protéagineuses riches en protéine}

Cette même analyse a réveillé que les graines de Ricinodendron heudelotii sont protéo-oléagineuse. Les feuilles de Myrianthus arboreus sont protéagineuses et les fruits de Treculia africana riches en sucres. Les graines de Irvingia gabonensis et de Sesamum indicum ont une faible teneur en eau. Cette faible teneur conclue que les graines de ces espèces peuvent être conservées pendant longtemps sans grand risque de contamination microbienne, contrairement aux feuilles de Myrianthus arboreus qui accumulent une quantité importante en eau. $\mathrm{Au}$ vu de ces résultats et compte tenu des valeurs nutritives des organes des plantes analysées, il est important de les consommer pour des raisons nutritionnelles. Les graines de Ricinodendron heudelotii et les feuilles de Myrianthus arboreus pauvres en sucres doivent être conseillées aux diabétiques. À l'opposé, les fruits de Treculia africana doivent être interdits aux diabétiques. Les feuilles de Myrianthus arboreus et les fruits de Treculia africana contiennent des quantités importantes de teneur en protéines. Les protéines sont les substances indispensables au bon fonctionnement de l'organisme car elles sont utilisées par celui-ci dans l'élaboration et le renouvellement des tissus et des

\section{BIBLIOGRAPHIE}

Agbo N. G., Uebersax M. \& Hosfield G., 1986. An efficient extraction technique of sugars from dry edible beans (Phaseolus vulgaris L.) estimation and H.P.L.C. University National of Côte d'Ivoire - Annals Series C Sciences 21 : 169 - 184.

Ake-Assi L. \& Boni D., 1990. Développement agricole et protection de la forêt : quel avenir pour la forêt ivoirienne ? Compte rendu de la XIIeme réunion plénière de l'AETFAT Symposium II : pp 169176. repas familiaux et apportent aux familles un équilibre alimentaire. Ces fruits ont des teneurs en sucres importantes qui peuvent être déconseillée aux diabétiques

cellules. Les résultats de cette analyse ont permis de lever une équivoque sur la teneur en protéines des échantillons des plantes utilisées. Les feuilles de Myrianthus arboreus, les graines de Strombosia pustulata, les graines de Beilschmiedia mannii et les graines de Treculia africana renferment le plus de protéines. II est d'intérêt de les consommer pour des raisons nutritionnelles et aussi pour résorber les éventuelles carences en protéines de l'organisme. Le protocole mis en place ne permet pas de mettre en évidence la qualité des protéines et des cendres si bien que d'autres études plus approfondies sont nécessaires. La comparaison des teneurs en cendres (matières minérales) des différentes plantes ont montré que Treculia africana a une teneur en cendres plus élevée que celle de Irvingia gabonensis et sont plus favorable à un apport de minéraux à l'organisme. Tout cela permette de dire que, aujourd'hui, en milieu rural, ces plantes consommées revêtent un grand intérêt pour la sécurité alimentaire des populations (consommation presque quotidienne de ces produits). En mettant en relief l'importance des différentes teneurs en matière grasse, en protéines, en cendres et en sucres des plantes spontanées alimentaires permet d'attirer l'attention des décideurs sur la cause de ces espèces (valorisation, sauvegarde). Les données sur la valeur nutritive d'un grand nombre de ces plantes restent encore inexistantes. Elles doivent faire l'objet d'une recherche nutritionnelle en vue de connaître leurs valeurs alimentaires.

A.O.A.C, 1975. Official Methods of Analysis (10 th). Association of official Agricultural Chemists, Washington D.C. Agron. Fr (1), $12 \mathrm{p}$.

B.i.p.e.a., 1976. Bureau interprofessionnel d'études analytiques, Recueil de méthodes d'analyses des communautés européennes.

Aubreville A., 1959. La flore forestière de la Côte d'Ivoire, Centre Technique Forestier Tropical, Nogentsur-Marne, France, 3 Tomes, pp. 322367Busson F., 1965. Plantes alimentaires de I'Ouest Africain. Étude botanique, biologique et 
chimique. Ministère de la Recherche Scientifique et des Armées, Paris, $568 \mathrm{p}$.

Dubois M., MC Cowen L.D., Schotcht.J., Rebersp.A. \& Smithf., 1956. Anal. Chemen. 28, 250 p.

Kouakou N. F., 2002. Étude comparative des caractéristiques physico-chimiques de l'huile de Irvingia gabonensis à celle de l'huile de palme vendu au marché d'Abidjan. Rapport de Stage de B.T.S, option Contrôle qualité $28 p$.

Kouamé N.M.T., 2000. Contribution à l'étude des plantes spontanées alimentaires du Département d'Oumé (Côte d'lvoire). Mémoire de D.E.A d'Écologie tropicale (Option: Végétale). Université de Cocody-Abidjan, Côte d'Ivoire, 122 $\mathrm{p}$

N'dri P., 1986. Contribution à l'étude de quelques plantes alimentaires spontanées de la région de Divo (Côte d'Ivoire). Mémoire de D.E.A d'Écologie Tropicale, Option Végétale. Université Nationale de Côte d'Ivoire, Abidjan, $65 \mathrm{p}$.

Silou T., Biyoko S., Heron S., Tchapla A. \& Maloumbi M.G., 2004. Caractéristiques physico-chimiques et potentialités technologiques des amandes de Irvingia gabonensis. La Rivista Italiana Delle Sostanze. 81 (1) : 49-57.

Tapi A., 2003. Étude des caractéristiques physicochimiques de la graine de Ricinodendron heudelotii (APKI). Mémoire de DEA de STA à I'Université d'Abobo-Adjamé, Côte d'Ivoire, $40 \mathrm{p}$.

Tchiegang C., Aboubakar D., Kapseu C. \& Parmentier M., 2005. Optimisation de l'extraction de l'huile par pressage des amandes de Ricinodendron heudelotii Pierre ex Pax. Journal of Food Engineering, 68 (1) : 79-87.

Traoré S., 2005. Étude de quelques caractéristiques physiques et chimiques de l'amande de Irvingia gabonensis (Kplé). Mémoire de DEA de UFRSTA à l'Université d'Abobo Adjamé, Abidjan, Côte d'Ivoire, $46 \mathrm{p}$.

Joseph J.K., 1995. Physico-chemical attributes of wild mango (Irvingia gabonensis) seeds. Bioresource Technology, 53 (2): 179-181. 